\title{
Análisis comparativo de los modelos de creación de laboratorios virtuales de la Universidad Politécnica de Madrid (UPM) y la Universidad Estatal a Distancia (UNED)
}

\author{
Roy Aguilera Jinesta ${ }^{1}$, Yuri Vázquez Pérez² \\ 1. Universidad Estatal a Distancia, Mercedes de Montes de Oca, San José Costa Rica. Apartado \\ postal San José 474-2050. raguilera@uned.ac.cr \\ 2. Universidad Estatal a Distancia, Programa Electrónica Multimedial, San José Costa Rica. \\ yvazquez@uned.ac.cr
}

\section{RESUMEN}

En el siguiente artículo se expone una comparación de los procesos de elaboración de laboratorios virtuales de la Universidad Politécnica de Madrid (UPM) y de la Universidad Estatal a Distancia de Costa Rica (UNED). El análisis comparativo se basó en la observación y estudio de los procesos de creación de laboratorios virtuales tanto en la UPM como en la UNED. Además, se realizaron entrevistas a los actores técnicos y académicos involucrados en los procesos de laboratorios virtuales de ambas universidades. Para la comparación se consideran aspectos tales como: definición de laboratorio virtual, proceso de elaboración, alcance y acceso, sistemas informáticos y bases de datos vinculados al laboratorio, repositorio y el modelo teórico. Dentro de los resultados de la comparación se determina que los procesos de creación de los laboratorios tienen un componente administrativo más definido en la UNED que en la UPM, así como la cantidad de involucrados y trámites es mayor en la UNED que en la UPM. Otro hallazgo es la diferencia en la integración de los laboratorios, en el caso de la UNED se cuenta con un repositorio en su sitio web, mientras que en la UPM se generó una interfaz que simula el campus universitario y el usuario debe desplazarse en ese mundo virtual para acceder al laboratorio. En cuanto al uso de los laboratorios, también se encontraron diferencias, en la UNED estas herramientas educativas se emplean para que los estudiantes practiquen, pero no forman parte de una evaluación sumativa, es decir, no tienen valor en la nota final de la asignatura (curso), mientras que, en la UPM, algunos laboratorios de emplean en las clases presenciales para evaluar a los estudiantes.

Palabras claves: Virtualidad, educación a distancia, simulación, UNED, UPM,

\begin{abstract}
This article compares the virtual laboratories development processes at Universidad Politécnica de Madrid and at Universidad Estatal a Distancia of Costa Rica. The comparative analysis was based on process observations, interviews with university professionals during an internship at UPM and expert criteria of the professionals at UNED involved in the virtual laboratories building process. The comparation considered key aspects such as: virtual laboratory main concept, building process, reach y access of the laboratory, computer systems y data bases connected to the laboratory, repository and theorical model. As a result of the comparison, the authors determined that the building process of a virtual laboratory at UNED has a stronger and defined administrative protocols, more employees involve and more quantity of paperwork than at the UPM. Another difference is the integration of the laboratories, at UNED all products are put in a web repository, while in the UPM, a virtual world, built by themselves, is used. Finally, related to the use of the laboratories in the evaluation processes, there was a considerable difference, at UNED not laboratory was used for student's evaluation, instead they are used for practices, but at the UPM, some of the laboratories were used not only for practice, but to evaluate student's learning.
\end{abstract}

Key words: Virtuality, distance education, simulation, UNED, UPM, 


\section{Introducción}

El desarrollo y uso de laboratorios virtuales forma parte de la oferta académica de la Universidad Estatal a Distancia por lo que es responsabilidad de la institución buscar opciones de mejora constante para que los estudiantes dispongan de ambientes más precisos, reales y amigables en pro de su desarrollo educativo. Gracias a iniciativas de la Vicerrectoría Académica y al Plan de Mejoramiento Institucional, se realizó una pasantía a la Universidad Politécnica de Madrid para observar, conversar, analizar y experimentar de primera mano la creación y el uso de los laboratorios virtuales, considerando aspectos como los equipos de trabajo, la tecnología empleada, los tiempos de producción, la vinculación entre academia y personal técnico asesor y la implementación de los laboratorios en el proceso de aprendizaje.

Para este artículo divulgativo se centró el desarrollo en una comparación de los procesos de desarrollo de los laboratorios virtuales de la UPM de España y de la UNED de Costa Rica, así como el análisis de aplicación de un modelo de diseño de dos producciones de cada universidad. En cuanto al objeto de estudio, este se define como la comparación de los procesos de elaboración de laboratorios virtuales de la Universidad Politécnica de Madrid y la Universidad Estatal a Distancia de Costa Rica.

\section{Universidad Politécnica de Madrid (UPM)}

Según la reseña histórica del sitio web de la UPM (UPM, 2019), los orígenes de esta Universidad datan del siglo XVIII, cuando se crearon la mayoría de los centros para la enseñanza de la arquitectura e ingenierías en España. Sin embargo, fue hasta el año 1971 cuando se fundó oficialmente la Universidad Politécnica de Madrid (UPM).

Los primeros estudios ofertados se dieron en arquitectura, así como ingeniería naval y de minas, posteriormente, nacen las escuelas técnicas de ingeniería en: Telecomunicaciones, Aeronáutica y Topografía, Geodesia y Cartografía. Luego se concretarían las facultades de Ciencias de la Actividad Física y el Deporte-INEF y la de Informática.

Actualmente, la UPM cuenta con una oferta que brinda 52 titulaciones de grado, 67 másteres y 45 doctorados; así como 280 titulaciones relacionadas a formación continua, formación permanente y acuerdos de doble titulación. El cuerpo docente está conformado por 2887 profesores e investigadores de planta y 414 investigadores contratados. (UPM, 2019). La UPM también cuenta con diversos grupos de investigación e innovación tecnológica (200 grupos). En relación con investigación, se han generado 42 patentes de una cartera vigente de 530. (UPM, 2019).

Toda esta infraestructura y talento humano proporciona el soporte necesario para mantener funcionando un sistema de educación presencial que atiende a 37638 estudiantes de grados, maestrías y doctorado. (UPM, 2019).

De acuerdo con el informe de pasantía de Aguilera y Vázquez, la UMP utiliza una metodología presencial, donde los estudiantes asisten periódicamente a clases, y un docente expone los temas de estudio, realiza prácticas, aclara dudas y brinda detalles complementarios para la correcta 
construcción de conocimientos. Los estudiantes deben realizar sus tareas y proyectos fuera del salón de clase y deben aplicar las pruebas escritas y (laboratorios en forma presencial en las instalaciones que la universidad disponga para tal fin. (Aguilera y Vázquez, 2016).

Una de las actividades medulares en los procesos de aprendizaje son los laboratorios, donde los estudiantes deben evidenciar de forma práctica la comprensión de conocimientos, la aplicación de teorías, el seguimiento de procesos y el uso de equipo técnico. Los laboratorios cuentan con un ambiente controlado y con normas de seguridad y, con equipo y recurso humano especializado. (Aguilera y Vázquez, 2016).

La UPM cuenta con instalaciones, equipo técnico y recurso humano para llevar a cabo todas las actividades que se plantean en su propuesta educativa presencial. Sin embargo, con el desarrollo y vinculación de las Tecnologías de Información y Comunicación (TIC); se han dado iniciativas y puesto en marcha proyectos que se pueden caracterizar por su uso de metodologías a distancia como ejes fundamentales.

Estas iniciativas de nuevas herramientas se dieron desde el área académica, y tuvieron un incremento en las solicitudes de producciones de laboratorios virtuales, por tal razón, la administración universitaria creó una instancia para fomentar la aplicación de tecnologías de los procesos educativos, el Gabinete de Tele-Educación

(GATE).

Universidad Estatal a Distancia de Costa Rica (UNED)

La UNED se fundó en 1977 mediante la ley No. 6044 en el mandato del presidente Daniel Oduber Quirós. Esta fue la primera Universidad con modalidad a distancia en América Latina. (UNED,2018). La UNED se ha caracterizado por la implementación de medios de comunicación social y la tecnología para llevar a cabo su quehacer académico, esto se destaca en su misión la cual declara que se hará uso “de los diversos medios tecnológicos que permiten la interactividad, el aprendizaje independiente y una formación humanista, crítica, creativa y de compromiso con la sociedad y el medio ambiente", para llevar educación superior a todos los sectores sociales, pero sobre todo a los que por razones "económicas, sociales, geográficas, culturales, etarias, de discapacidad o de género, requieren oportunidades para una inserción real y equitativa en la sociedad" (UNED,2013).

Actualmente, la UNED cuenta con 86 carreras de grado distribuidas en cuatro Escuelas: Administración, Educación, Exactas y Naturales y Sociales. Además, se dispone de 24 maestrías y 4 doctorados. (CIEI-UNED,2018). En cuanto a investigación, la UNED trabaja en 26 nodos de investigación, que abarcan 215 proyectos. (CIEIUNED,2018).

Desde su creación la UNED utilizó el modelo de educación a distancia y actualmente se atiende a una población de más de 31370 estudiantes (CIEIUNED,2018). El modelo a distancia se ha caracterizado por el uso intensivo de elementos 
tecnológicos, medios de comunicación masivos, tal como lo establece su modelo pedagógico (UNED, 2005 , p.4) que estipula que para alcanzar sus objetivos uno de los derroteros es "Utilizar para ello, en forma intensiva, los medios de comunicación escritos, audiovisuales $\mathrm{y}$, en el presente, los informáticos e interactivos". Además, se le otorga al estudiante el control total de su espacio y tiempo de estudio, en especial en la nueva sociedad del conocimiento donde, según el modelo pedagógico de la UNED (UNED, 2005, p.6) "Se hace necesaria una concepción metodológica más abierta, más flexible, que ofrezca, al estudiante, herramientas para construir su propio proceso de enseñanza-aprendizaje y lo haga protagonista en la apropiación del conocimiento". Esto con la meta de cumplir con uno de los principios de educación de adultos el cual se basa en "aprender a aprender", el cual se enfoca en que el estudiante "sea capaz de realizar aprendizajes significativos por si solo en una amplia gama de situaciones y circunstancias" (UNED, 2005, p.11).

En este modelo se brindan apoyos tanto presenciales como virtuales para guiar al estudiante en su aprendizaje, como lo indica el CIEI en un estudio comparativo del 2017, dos de los espacios de interacción de los estudiantes de la UNED en su proceso de aprendizaje son las tutorías y los entornos virtuales (UNED, 2005, p.6).

\section{Laboratorios virtuales}

La Real Academia Española (RAE, 2019) define laboratorio como el "lugar dotado de los medios necesarios para realizar investigaciones, experimentos y trabajos de carácter científico o técnico". La conceptualización anterior, aunque no lo manifiesta de forma explícita, se toma para un laboratorio físico, es decir con estructura palpable en un espacio. Estos ambientes pueden llegar a ser muy costosos, tanto por el costo deconstrucción, como por el mantenimiento y equipamiento de laboratorio y seguridad. Adicionalmente se encuentran limitados a una capacidad máxima y un horario especifico. Es en esta búsqueda donde Las TIC llegan a reducir sensiblemente estos costos, ya que permiten crear laboratorios virtuales que complementan o sustituyen, en algunos casos, los laboratorios físicos. Un laboratorio virtual es una herramienta educativa apoyada por las TIC, autores como Becerra et al. (2016) relacionan estas herramientas con simulaciones computacionales que permiten a los estudiantes interactuar con sistemas informáticos dinámicos.

Los laboratorios virtuales se utilizan en diversas áreas del conocimiento (p.e., física, química, informática, matemática, biología, estadística, educación, administración, contabilidad, entre otras). Rodríguez et al. (2014) ponen de manifiesto la versatilidad y utilidad de los laboratorios virtuales, destacando la preparación del estudiante en el laboratorio virtual, previo a la práctica en un laboratorio físico. Además, expresan que los laboratorios virtuales "permiten, de una manera eficiente y rápida, simular y analizar muchas variantes del fenómeno objeto de estudio”.

Es importante aclarar que un laboratorio virtual no es lo mismo que uno remoto. Este último se define como un sistema que integra un componente virtual y otro físico. La Escuela de Posgrado y Educación Continua (EPEC) de la Universidad del Rosario en Argentina citado por Salas et al. (2017) señala que la parte virtual es "la interfaz de usuario encargado 
de controlar las acciones" y la parte física es la que ejecuta la acción solicitada. En el caso de los laboratorios virtuales el componente físico es completamente ausente e innecesario.

Según Sánchez et al (2017) en su Modelo para el Diseño de Laboratorios Virtuales, un laboratorio virtual se entiende como:

El conjunto de estrategias y actividades mediadas pedagógicamente que al usar simulaciones, videos $\mathrm{u}$ otros recursos multimedia (por medio de las tecnologías de la información y comunicación), propician el alcance de los objetivos de aprendizaje propuestos, dentro de un ambiente interactivo y colaborativo de los estudiantes. (Sánchez et al, 2017, p.5).

Un laboratorio virtual debe tener componentes teóricos, procedimentales, experimentales y de tareas, la definición anterior se complementa con la siguiente: ambiente de aprendizaje digital que reúne la teoría, el procedimiento, un factor experiencial, la asignación y la evaluación de los objetivos propuestos. Donde es posible aplicar el conocimiento teórico en una situación práctica a través de la mediación pedagógica y del uso de las Tecnologías de Información y Comunicación (TIC)" (Sánchez et al, 2017, p.5).

Para que un laboratorio virtual tenga una integración exitosa debe contener los siguientes elementos: teoría, procedimiento, factor experiencial, asignaciones y referencias. También se consideran otros componentes opcionales como lo son actividades de autoevaluación y guías de mediación para estudiantes y docentes. (Sánchez et al, 2017, p.13)
El modelo de Sánchez et al. (2017) también considera los siguientes elementos técnicos: dispositivos de interacción, el diseño de interfaz, la colaboración y la interacción. Estos elementos se clasifican en una escala de 1 a 4, donde cada incremento representa un nivel más de complejidad en el elemento respectivo.

\section{Contexto Metodológico}

\section{Modelo de creación de laboratorios virtuales}

Con el creciente desarrollo de las herramientas TIC se ha fortalecido la creación e implementación de los laboratorios virtuales en el ámbito educativo, pero este desarrollo no puede estar al margen de normas de control y sistematización, por lo que las instituciones han optado por organizar mediante modelos la creación, modificación y, en algunos casos, la implementación en el entorno educativo de los laboratorios virtuales.

En la UNED existe un trámite oficial por el cual se inician, desarrollan y finalizan los procesos de creación de laboratorios virtuales. Este modelo es en el que se basará el análisis entre la UPM y la UNED, el cual será explicado posteriormente.

\section{Comparación de creación de laboratorios virtuales entre UPM y UNED}

Para la comparación del proceso de creación de laboratorios virtuales se analizarán laboratorios de cada una de las casas de enseñanza, considerando los siguientes elementos vinculados al proceso de producción:

- Instancias universitarias encargadas de la producción.

- Personal de producción. 
- Proceso de creación.

- Características de los laboratorios virtuales.

$$
\begin{array}{ll}
\circ & \text { Repositorio de los laboratorios. } \\
\circ & \text { Alcance y acceso al laboratorio. } \\
\circ & \text { Sistemas informáticos } \\
& \text { enlazados y bases de datos. } \\
& \text { Modelo teórico. }
\end{array}
$$

\section{Resultados}

\section{Personal de producción}

A continuación, se brinda una comparación entre la cantidad de funcionarios y su rol dentro del proceso de desarrollo de un laboratorio virtual de la UPM y la UNED (Tabla 1).

Tabla 1. Personal involucrado en la producción de laboratorios.

\begin{tabular}{lll}
\hline Rubro & UPM & UNED \\
\hline $\begin{array}{l}\text { Cantidad de diseñadores } \\
\text { gráficos. }\end{array}$ & 1 & 1 \\
$\begin{array}{l}\text { Cantidad de } \\
\text { programadores }\end{array}$ & 1 & 1 \\
$\begin{array}{l}\text { Cantidad de académicos } \\
\text { involucrados }\end{array}$ & De 2 a 4 & 3 \\
\hline
\end{tabular}

Para la producción de laboratorios virtuales la UPM cuenta con el GATE, mientras que en la UNED la Dirección de Producción de Material Didáctico (DPMD) es la entidad encargada de la misma tarea. A continuación, se presenta un análisis comparativo entre estas instancias (Tabla 2).

\section{Comparación entre personal de producción}

Una característica en común de las producciones en cuanto a laboratorios virtuales de la UNED y de la UPM es el trabajo con la contraparte académica; ya que, en ambos casos, se cuenta con un profesional que supervisa los aspectos teóricos de la temática que trata el laboratorio, asimismo, sus apreciaciones y recomendaciones son vinculantes y se consideran durante todo el proceso de producción.

En la UPM la tarea de revisión temática recae sobre uno o varios profesionales, en los laboratorios analizados se aprecia que hay un equipo docente vinculado al laboratorio de 2 personas y en otro caso el equipo es de 4 profesionales.

En la UNED este proceso se encuentra conformado por tres figuras: un especialista de contenidos, un revisor de contenidos y el encargado de cátedra. El especialista de contenido es el encargado de aportar el material teórico que va a ser incluido en la producción, además de trabajar en coordinación con el equipo de programación del PEM a cargo del desarrollo. El revisor de contenido es un experto en la materia y su función es ser la contraparte revisora del contenido aportado por el especialista. Encargado de cátedra, también puede aportar su punto de vista técnico en los elementos temáticos del laboratorio y es el encargado de dar la aprobación final del contenido para pasar a la etapa de desarrollo, en muchas ocasiones el rol de revisor de contenido es interpretado por el encargado de cátedra por lo que se emplean dos personas en vez de tres para la parte académica. 
Tabla 2. Comparación entre las instancias GATE y DPMD

\begin{tabular}{|c|c|c|}
\hline $\begin{array}{l}\text { Elemento de } \\
\text { comparación }\end{array}$ & GATE & DPMD \\
\hline $\begin{array}{llll}\text { Instancia } & \text { a } & \text { la } & \text { que } \\
\text { pertenece } & & & \\
\end{array}$ & $\begin{array}{l}\text { Vicerrectorado de Planificación } \\
\text { Académica y Doctorado. }\end{array}$ & Vicerrectoría Académica \\
\hline Líneas de trabajo & 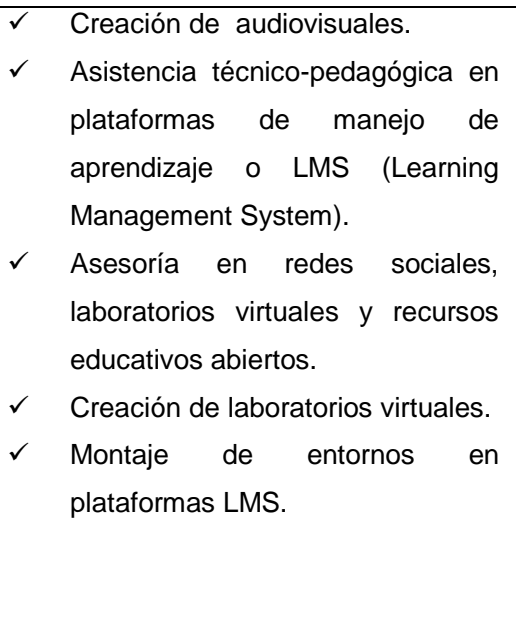 & $\begin{array}{l}\checkmark \quad \text { Creación de audiovisuales. } \\
\checkmark \quad \text { Asistencia técnico-pedagógica en } \\
\text { plataformas de manejo de aprendizaje o } \\
\text { LMS (Learning Management System). } \\
\checkmark \quad \text { Creación y asesoría en videoconferencias. } \\
\checkmark \quad \text { Asesoría al personal académico para la } \\
\text { producción de material didáctico escrito: } \\
\text { libros, revistas, guías de estudio, material } \\
\text { complementario y antologías. } \\
\text { Creación y asesoría en la producción de } \\
\text { multimedios: animaciones digitales, } \\
\text { laboratorios virtuales, diseños gráficos y } \\
\text { vídeos interactivos. }\end{array}$ \\
\hline
\end{tabular}

\begin{tabular}{|c|c|c|c|c|}
\hline $\begin{array}{l}\text { Grupo de trabajo exclusivo } \\
\text { para laboratorios virtuales }\end{array}$ & Si. & & No. & \\
\hline $\begin{array}{l}\text { Subinstancias que la } \\
\text { componen }\end{array}$ & & $\begin{array}{l}\text { Grupo de trabajo general. } \\
\text { Grupo de trabajo exclusivo de } \\
\text { laboratorios virtuales. }\end{array}$ & $\begin{array}{l}\checkmark \checkmark \\
\checkmark \\
\checkmark\end{array}$ & $\begin{array}{l}\text { Programa de Producción de Material } \\
\text { Audiovisual (PPMA). } \\
\text { Programa de Aprendizaje en Línea (PAL). } \\
\text { Programa de Videoconferencia y } \\
\text { Audiográfica (PVAU). } \\
\text { Programa de Material Didáctico Escrito } \\
\text { (PROMADE). } \\
\text { Programa Electrónico Multimedial (PEM). }\end{array}$ \\
\hline
\end{tabular}




\section{Proceso de creación de un laboratorio virtual}

A continuación, en las figuras 1 y 2 , se refleja el proceso de creación de un laboratorio virtual en cada una de las universidades. Según las figuras 1 y 2 , el proceso de creación de un laboratorio virtual en ambas universidades es muy similar, se inicia con una solicitud de una instancia académica a la parte encargada de la producción del laboratorio, ya sea el GATE, en la UPM, o el PEM, en la UNED. Luego de llenar un formulario se realiza una reunión entre el personal académico interesado en un laboratorio virtual y los funcionarios (del GATE o PEM) asignados a la producción.

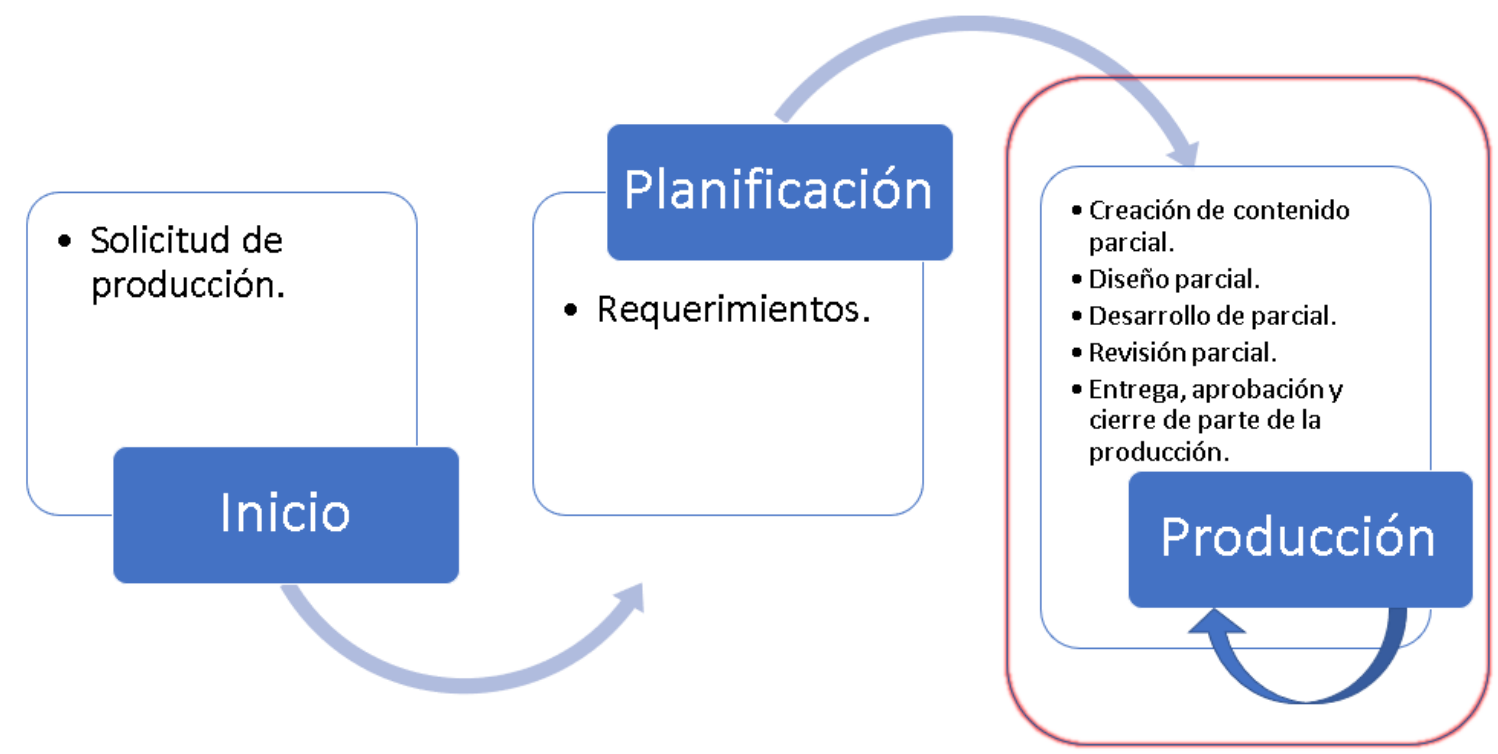

Figura 1: Proceso de producción de un laboratorio virtual en la UPM. Fuente: creación propia. 2019

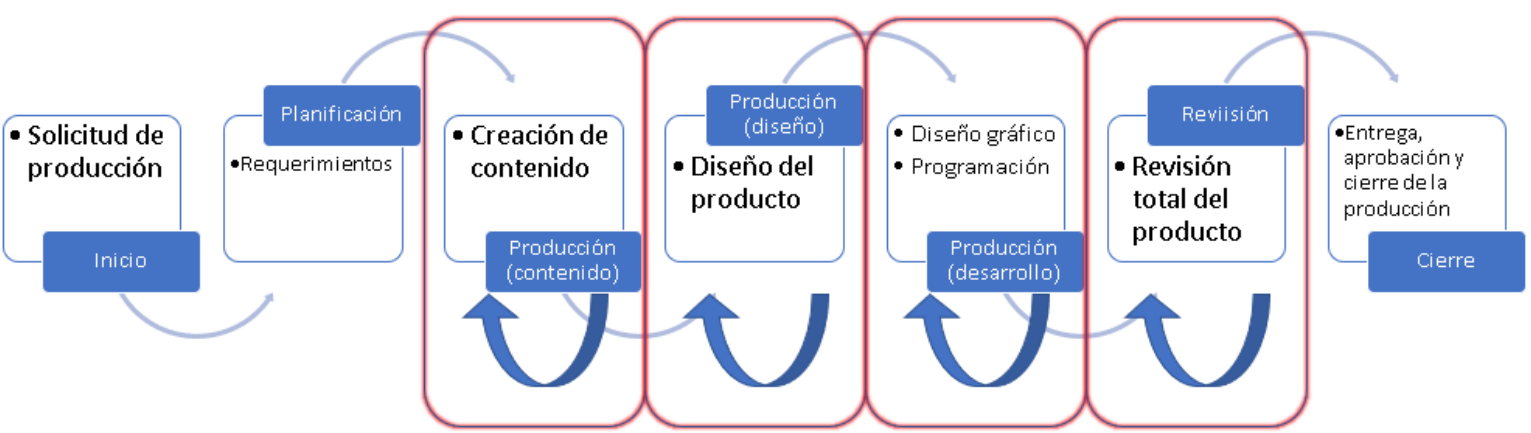

Figura 2: Proceso de producción de un laboratorio virtual en la UNED Fuente: creación propia. 2019

En esa primera reunión, se analizan los requerimientos de usuario en relación con: lo que se desea que haga el laboratorio, el nivel de detalle del entorno virtual, exclusividad en cuanto a imágenes (nuevas o prediseñadas), necesidad de desarrollar una aplicación externa y vinculada al laboratorio, alojamiento, descripción y cantidad de las prácticas que se realizarán y acciones complementarias que 
debe realizar el estudiante al momento de utilizar el laboratorio.

Una vez alcanzado el acuerdo entre las partes se inicia la creación del laboratorio virtual. En la UPM la etapa de elaboración de contenidos va al mismo ritmo que la de desarrollo y recae sobre el profesor que realiza la solicitud. En la UNED, este proceso difiere, primero se realiza una etapa de producción de contenidos y una vez finalizada y aprobada esta etapa es que se pasa a la fase de diseño y desarrollo del laboratorio.

Este desarrollo de contenido se realiza entre el especialista de contenido asignado por la cátedra y el productor académico del PEM, una vez finalizada la creación de contenidos, se remite el material al revisor de contenidos para que haga sus observaciones y finalmente al encargado de cátedra para la aprobación definitiva del contenido.

Con el contenido aprobado, se prosigue con la etapa de desarrollo, en esta intervienen tanto el personal programador y diseñador del PEM como el personal académico de la instancia solicitante del producto, es en esta fase donde se podrían realizar cambios menores del contenido si fuera necesario; pero nunca cambios significativos, si por cualquier eventualidad habría que cambiar el contenido a profundidad, se debe cancelar la producción y solicitar una nueva con su correspondiente etapa de creación de contenido.

Finalmente, se hace entrega del producto terminado, se hace una revisión final y se da por aceptado el nuevo laboratorio virtual, el mismo se alojará en el servidor determinado en los requerimientos.

\section{Características de los laboratorios virtuales}

De acuerdo con las observaciones efectuadas durante la pasantía a la UPM, entrevistas realizadas al personal del GATE y profesionales académicos, así como con observaciones detalladas a los procesos de producción en el PEM de la UNED se detallan algunas características importantes detectadas en los laboratorios virtuales. Estas características se enfocan en cinco aspectos: repositorio de los laboratorios, alcance del laboratorio, acceso al laboratorio, sistemas informáticos y bases de datos enlazadas al laboratorio y modelo teórico del laboratorio.

En la figura 3, se evidencian las características de los laboratorios de la UPM y la UNED.

A continuación, se describirán las características de los laboratorios, tanto de la UPM como de la UNED.

\section{Repositorio de los laboratorios}

En la UPM, todos los laboratorios virtuales están enlazados en un solo mundo virtual, es como una gran comunidad donde se desarrolla cada espacio para un laboratorio de forma organizada. $\mathrm{Al}$ estar en dicho mundo, el estudiante puede visitar otros laboratorios o simplemente pasear por los diferentes ambientes que componen este universo. 


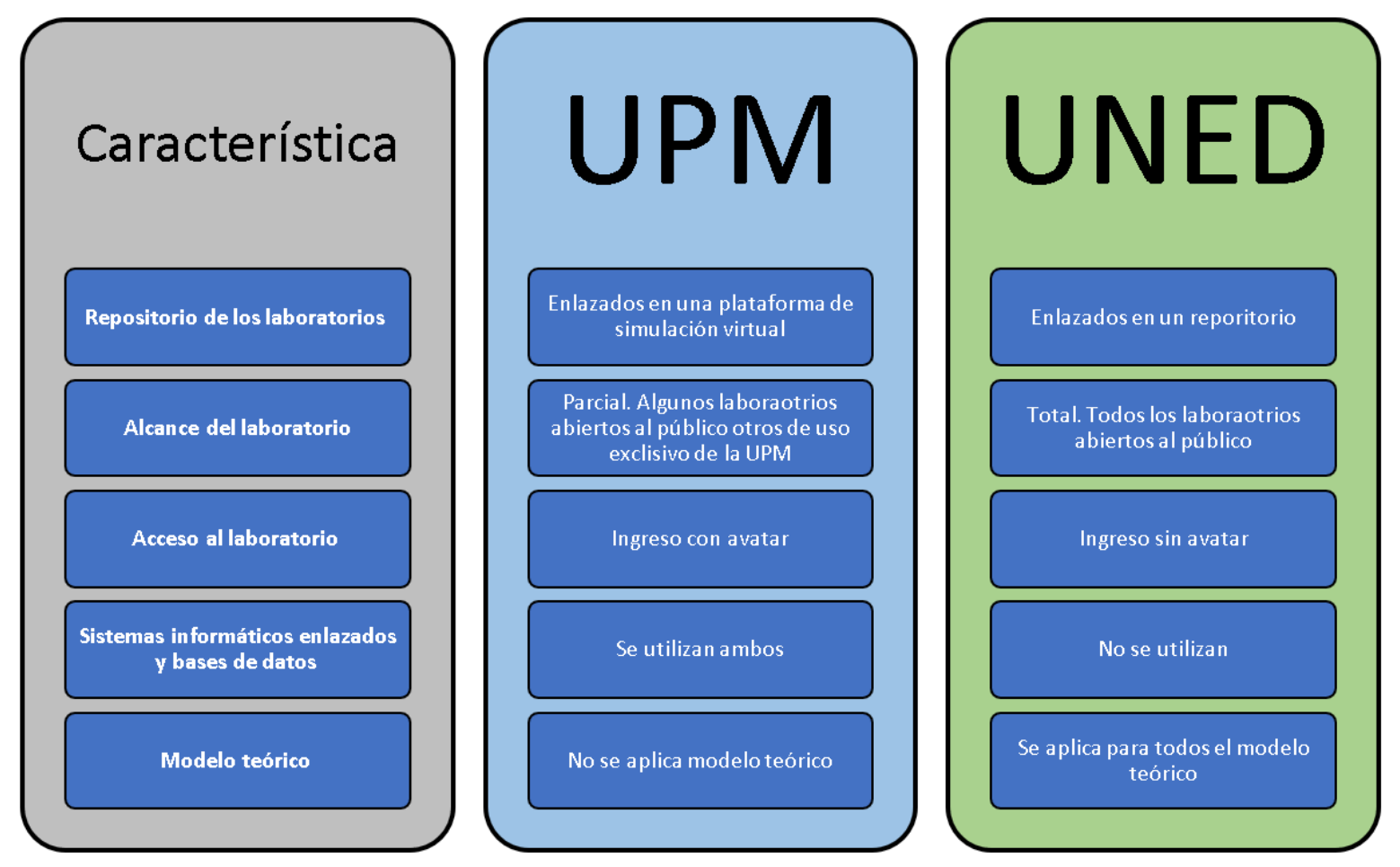

Figura 3: Comparación de las características de los laboratorios virtuales UPM-UNED

Fuente: creación propia. 2019

En la UNED, los laboratorios son completamente individuales, es decir, no están inmersos en un mundo virtual. Todos los productos del PEM están contenidos en un solo repositorio donde se puede tener acceso a todos los laboratorios por medio de enlaces ubicados en la página web de la universidad.

\section{Alcance del laboratorio}

Con respecto al alcance, la UPM posee laboratorios abiertos a toda la comunidad ya sea universitaria o no, y otros que son de uso exclusivo de las asignaturas a las cuáles brinda servicio el laboratorio.

Por su parte, la UNED tiene abierto al público todos sus laboratorios, por lo que cualquier persona alrededor del mundo, con conexión a Internet, puede ingresar al repositorio y utilizar los laboratorios.

\section{Acceso al laboratorio}

En cuanto al acceso, en la UPM, todos los usuarios que ingresan a los laboratorios lo hacen por medio de un sistema de simulación de la realidad, también llamado realidad o mundo virtual. En este mundo virtual, los usuarios deben crear un usuario animado el cual semeja a una caricatura, a esto se le denomina avatar.

Con el avatar, el usuario camina en el mundo virtual y puede ingresar a los distintos laboratorios 
que tenga disponibles y acceso permitido.

En la UNED, el usuario ingresa a la página web de la universidad y accede directamente a los laboratorios, sin necesidad de un avatar. En algunos laboratorios, se solicita que el usuario digite un nombre para que durante el uso del producto se despliegue ese dato y personalizar los mensajes que se le brindan, este nombre se usa de manera temporal en el laboratorio, por lo que si el usuario ingresa de nuevo lo puede cambiar.

\section{Sistemas informáticos $y$ bases de datos enlazadas al laboratorio}

En la UPM, se cuenta con sistemas informáticos enlazados con los laboratorios virtuales; para algunos laboratorios, por razones de organización de la asignatura y la cantidad de estudiantes, se generó una aplicación de reserva de espacio para su uso. El estudiante ingresa a su entorno virtual y en una pizarra hace su reserva de espacio para practicar.

Además, la mayoría de los laboratorios virtuales tienen un respaldo de la actividad estudiantil en bases de datos externas. Esto lo utilizan los docentes para calificar y realizar evaluaciones formativas.

En la UNED, ningún laboratorio posee enlaces a una aplicación externa. En referencia a la vinculación y uso de bases de datos, hasta el momento ningún laboratorio cuenta con esta característica, esto debido a que los datos generados por los estudiantes en los laboratorios se borran cuando se cierra el mismo.

\section{Modelo teórico del laboratorio}

En la UPM, la elaboración de laboratorios virtuales se rige por los aportes del equipo técnico (programación y diseño) del GATE y por las necesidades que la parte académica estipula, por lo que la estructura en cuanto a elementos que debe tener el producto depende de esos actores y no de una línea teórica base que guía la calidad y completitud del laboratorio, esto genera que todos los laboratorios sean completamente diferentes en cuanto a sus elementos de instrucción teórica, mediación didáctica, práctica y tipos de evaluación.

En la UNED, los laboratorios creados a partir del 2016 responden al modelo desarrollado por Sánchez et al, en el año 2017, aunque el modelo se publicó en ese año, ya desde el 2016 se dieron iniciativas para implementarlo de forma parcial en las creaciones de laboratorios virtuales. Esto hace que en la UNED se cuente con un modelo que guía la estructura y elementos didácticos de los laboratorios. Define qué partes debe tener una producción para que se considere un laboratorio virtual, establece el orden de dichas partes, propone posibilidades de actividades de práctica y evaluaciones.

\section{Discusión}

En cuanto a las instancias universitarias vinculadas a la creación de laboratorios virtuales, ambas universidades tienen definidos departamentos especializados adscritos al área académica, por lo que los servicios que prestan se enfocan en asesorar y crear productos con miras a ser empleados en procesos educativos. 
Sin embargo, en la UPM, y dentro del GATE, hay un grupo exclusivo para el trabajo con laboratorios virtuales, mientras que, en la UNED, el PEM no solo se avoca a estos productos, sino que debe también crear animaciones, diseños gráficos, vídeos y otros elementos que no son para laboratorios virtuales. Esta situación hace que el personal de la UPM disponga de más tiempo para explorar nuevas opciones en cuanto a mejoras de sus laboratorios y hacer propuestas al área académica. En contraparte, en la UNED, el personal del PEM no dispone de tiempo más que para hacer las producciones que tienen a cargo, por lo que el espacio para la investigación y exploración de mejoras se ve drásticamente reducido en comparación con la UPM.

Otro aspecto muy importante con respecto al personal involucrado en una producción de un laboratorio virtual es el relacionado a la parte académica, en la UPM intervienen en cada laboratorio de 2 a 4 profesores, quienes forman parte del diseño y evalúan el laboratorio en sus elementos teóricos. En la UNED, a pesar de que se reportan 3 académicos, no todos tienen las mismas funciones, uno funge como responsable del contenido y los otros dos se limitan a evaluar el producto $\mathrm{y}$ hacer recomendaciones, pero no necesariamente con un perfil para mejora la parte teórica de la temática que se está tratando en el laboratorio, uno de esos dos profesionales se enfoca principalmente en aspectos administrativos de la producción, como dar vistos buenos en diversas etapas, pero se basa en lo que dictaminen los otros dos profesionales académicos. Estas diferencias hacen que los laboratorios en la UPM tengan una evaluación teórica más amplia que en la UNED, ya que se dispone de un mayor número de puntos de vista y análisis de la temática.

En lo que respecta al proceso de producción, en ambas universidades se establece un procedimiento claro que debe seguir quien desee un laboratorio virtual, en la UPM, el proceso de resume en tres etapas, siendo la tercera un ciclo con diversas etapas y que se repite hasta que se termina la producción. El proceso en la UNED es más seccionado, ya que contempla fases dedicadas a aspectos muy puntuales en la producción.

La diferencia en la tercera etapa del proceso de la UPM y en el grupo de etapas 3, 4, 5, 6, y 7 de la UNED, hace que la producción en la universidad europea tenga un desarrollo en paralelo de sus diversos componentes, es decir, tanto la creación de contenido, como el diseño y desarrollo en cuanto a programación del laboratorio se hacen al mismo tiempo, pero dividido en subtemas dentro del mismo laboratorio.

En la UNED el proceso de creación de contenido se hace en su totalidad para todo el laboratorio antes de iniciar la fase de diseño y de desarrollo programado.

Esto da como consecuencia que en la UPM los posibles cambios que surjan durante la producción del laboratorio se puedan hacer de forma más expedita y sin afectar las demás partes que de todas formas no se han iniciado aún, así, las lecciones aprendidas y mejoras de los primeros pasos del laboratorio se aplican en las siguientes. En la UNED, el proceso para cambios es más rígido, ya que si en la etapa 4 (diseño) se detectan errores que impliquen cambios en la etapa de contenidos se debe volver a revisar en su totalidad, generando 
atrasos considerables en la finalización del producto. Las características de los laboratorios son aspectos muy importantes en los procesos de producción, ya que pueden tornar su creación sencilla o compleja, así como alterar los tiempos de entrega de los productos. Para ambas universidades se analizaron las mismas características y se evidenciaron diferencias significativas que se discutirán a continuación.

Los repositorios de los laboratorios en la UPM tienen una presentación mucho más interactiva de sus homólogos en la UNED, ya que al estar inmersos en un mundo virtual y requerir una representación gráfica (avatar) por parte del usuario, quien controla el desplazamiento y movimientos del avatar, resulta más atractivo el ingresar a un laboratorio, inclusive si no se ingresara a ningún ambiente de trabajo (laboratorio) el solo hecho de caminar por el mundo virtual ofrece a los visitantes la oportunidad de explorar. En la UNED el usuario no dispone de ese grado de interactividad ni desarrollo visual, ya que los laboratorios están en un repositorio en el sitio web institucional, omitiendo oportunidades para incentivar el uso de estos recursos.

Sin embargo, hay otra característica que en los laboratorios de la UNED se presenta de forma masiva y no así en los de la UPM, el alcance. En la UNED, los laboratorios están disponibles para todo el usuario que desee visitarlos, sin distinción de si se es estudiante o no o si reside o no en el territorio nacional. Siempre que se cuente con una computadora con acceso a Internet se puede utilizar cualquier laboratorio de la universidad. En la UPM, el acceso libre está limitado a unos pocos laboratorios, lo que provoca que este recurso no sea accesible para todo público. Esta diferencia evidencia uno de los postulados principales de la UNED, el cual es la democratización de la educación superior, además, de su vocación de institución con un alto componente de acción social; con respecto a esos elementos y la UPM, no es que no los tenga, pero al menos no se pueden evidenciar en sus laboratorios virtuales, y la razón es muy válida y se relaciona directamente con la siguiente característica: los sistemas informáticos y bases de datos enlazadas a los laboratorios.

En cuanto al enlace de sistemas informáticos y bases de datos, los laboratorios de la UPM cuentan con diversos sistemas enlazados, además, la mecánica de trabajo en los procesos educativos que emplean laboratorios requieren que se almacenen en bases de datos cierta información que producen los estudiantes mientras trabajan en los ambientes virtuales, esto limita el uso de algunos laboratorios a solo estudiantes de la universidad, ya que si se abre el acceso a otras personas se generarían datos que dificultan la examinación por parte de los docentes. En el caso de la UNED ningún laboratorio emplea conexión a bases de datos ni requiere el apoyo de sistema informáticos externos, por lo que no existe la posibilidad de almacenar en una base de datos la información generada por los estudiantes producto de su trabajo en los laboratorios.

Finalmente, y en referencia al modelo teórico empleado en los laboratorios, los productos de la UPM no siguen modelo alguno, sino que las características, metodología o actividades que debe tener un producto de este tipo son establecidos por los integrantes de cada equipo de trabajo de acuerdo con su experiencia y criterio profesional. 
Por su parte, los laboratorios de la UNED siguen el modelo teórico propuesto por Sanchéz et al (2017), donde todos los productos deben cumplir con los elementos mínimos y opcionalmente con algunos otros aspectos que son complementarios. Esta gran diferencia hace que el proceso de creación en la UNED tenga un parámetro claro de lo que se desea en cuanto de un laboratorio virtual, facilitando así su evaluación para posibles ajustes que se deben dar con la intención de respetar el modelo teórico.

\section{CONCLUSIONES}

El desarrollo de laboratorios virtuales tanto en la UPM como en la UNED es un trabajo integrado entre la parte académica y la parte de asesoría técnica, esto evidencia, en ambas instituciones, un trabajo integrado y un procedimiento claro para la producción de material didáctico.

Esta metodología de trabajo promueve la labor colaborativa entre profesionales de diversas áreas, en la UPM el equipo de desarrollo es más reducido que en la UNED. Esto genera que el desarrollo del laboratorio virtual sea más rápido en la institución europea que en la latina, pero la visión del material temático también es reducida, al contar con solo una persona que revisa el contenido, en la UNED a pesar de que se invierte más tiempo en finalizar el producto, el contenido lleva una riqueza amplia, debido a los aportes de dos o tres profesionales diferentes y se cuenta con un filtro más estricto en la inclusión de los contenidos al laboratorio virtual.

A pesar de que la UNED parece más exclusiva en cuanto a los contenidos de sus laboratorios, no aventaja a la UPM, ya que al contar ambas con revisión por parte de profesionales el contenido es válido, pero en la UMP la producción de un laboratorio no tarda tanto como en la UNED, en parte, esto se debe a las revisiones excesivas que se aplican en la UNED y que al final atrasan la producción. Si se elige adecuadamente al especialista en contenido las revisiones no deben superar un equipo de dos personas.

El acceso a los laboratorios varía entre las dos universidades, mientras en la UPM, el acceso a los laboratorios virtuales se realiza por medio de un usuario y una contraseña, en la UNED, el acceso está abierto a toda la población. Esto último facilita a la población, ya sea estudiantil o no, el uso de los laboratorios, pero al no solicitar datos de ingreso se genera una desventaja, ya que no se tiene información de contacto para futuras consultas a los usuarios de los laboratorios, ni un perfil de quien está utilizando este recurso didáctico.

Con respecto a la configuración del equipo; en la UPM, la utilización de los laboratorios virtuales requiere de una configuración previa de la computadora donde se utilizará y la solicitud de un usuario y una contraseña al sistema de la universidad. Mientras que, en la UNED, los laboratorios virtuales se pueden acceder desde cualquier dispositivo móvil y no requiere configuración previa, con lo que se facilita su uso en comparación con los de la UPM.

En cuanto a la generalidad del proceso de producción, se concluye que en la UPM existe un desarrollo más flexible, rápido y dinámico, sin embargo, carece de algunos aspectos de fundamentación teórica que acercan a los laboratorios a una imagen más controlada en cuanto a control didáctico y de mediación. 
Estas características en el proceso productivo, obedece al modelo educativo, ya que en la UNED cada material didáctico se centra en que el estudiante estudie y tenga la oportunidad de autoevaluar lo aprendido en un mismo material, mientras que en la UPM los docentes encargados del laboratorio específico son los que definen si el material tendrá elementos de contenidos o si será únicamente para practicar algún tema que se estudia en la clase presencial.

A pesar de la seguridad teórica que en la UNED se le otorga a los laboratorios virtuales, se deben hacer mejoras en el proceso de desarrollo, ya que muchas veces las mismas revisiones atrasan la finalización del producto.

\section{AGRADECIMIENTOS}

Se agradece a las y los integrantes de la Comisión de Laboratorios Virtuales de la UNED por su apoyo.

También se agradece al equipo técnico del GATE de la Universidad Politécnica de Madrid y en especial al su director el señor Luis Catalán, quienes colaboraron y abrieron sus puertas para compartir su experiencia en el desarrollo de laboratorios virtuales.

\section{Referencias}

Aguilera, R. Vázquez, Y. (2016). Informe de Pasantía a la Universidad Politécnica de Madrid. UNED.

Becerra, D. Mora, C. Ordóñez, A. Sánchez, R. (2016). Enseñanza de la Ley de Ohm utilizando laboratorios virtuales con estudiantes de ingenierías de la Universidad Antonio Nariño. Latin-American Journal of Physics Education, volumen (10), , Latin-American Journal of Physics Education, 2016, Vol.10(4), 4304-1-4304-4.

Centro de Investigación y Evaluación Institucional CIEIUNED. (2017). Estudio comparativo entre el Modelo Pedagógico de la Universidad Estatal a Distancia, Costa Rica y el Modelo de Comunidades de Investigación (Community Inquiry) de Garrison y colaboradores. Recuperado de: https://www.uned.ac.cr/viplan/images/ciei/INVESTIGA CIONES_2017/AGOSTO_2017/AGOSTO_2017/infor $\underline{\text { me_final_de_Jorge.pdf }}$

Centro de Investigación y Evaluación Institucional CIEIUNED. (2018). Anuario Estadístico 2017. Recuperado de:

https://www.uned.ac.cr/viplan/images/ciei/ANUARIO_ 2017/Anuario 2017 versi\%C3\%B3n final 19-05-

$\underline{18 . p d f}$

Real Academia Española. (2019). Definición de Laboratorio. Recuperado de: https://dle.rae.es/?id=MjESnb2

Rodríguez, Y. Molina, V. Martínez, M. Molina, J. (2014). El Proceso Enseñanza-Aprendizaje de la Química General con el Empleo de Laboratorios Virtuales. Revista Avances en Ciencias e Ingeniería. Recuperado de:

file:///C:/Users/royaj/Downloads/Dialnet-

ElProcesoEnsenanzaaprendizajeDeLaQuimicaGeneralC on-4737918.pdf

Sánchez, M. Salas, I. Berrocal, V. Arias, P. Vázquez, Y. (2017). Modelo para el diseño de laboratorios virtuales para la Universidad Estatal a Distancia. UNED.

UPM. (2019). Reseña Histórica. Recuperado de: https://www.upm.es/UPM/Historia/ResenaHistorica

UMP. (2019). UPM en cifras. Recuperado de: https://www.upm.es/sfs/Rectorado/Gabinete\%20del\%2 0Rector/Notas\%20de\%20Prensa/2019/07/documentos/ UPM\%20en\%20cifrasCALIDAD\%202019.pdf 
UNED. (2005). El modelo Pedagógico. Vicerrectoría Académica. San José, Costa Rica. Recuperado de: https://www.uned.ac.cr/academica/images/igesca/materi $\underline{\text { ales/24.pdf }}$

UNED. (2013). Misión de la UNED. Recuperado de: https://www.uned.ac.cr/rectoria/myv/19-rectoria/118$\underline{\text { mision-de-la-uned }}$

UNED. (2018). Reseña histórica. Recuperado de: https://www.uned.ac.cr/29-auditoriainterna/infgeneral/76-resena-historica. 